\title{
Kidney Pathology and Outcomes in ANCA-Associated Vasculitis: Retrospective Analysis of 85 Patients
}

\author{
Elena Zakharova ${ }^{1,2, *(\mathbb{D})}$, Anastasiia Zykova ${ }^{1,3}$, Tatyana Makarova ${ }^{1}$, Eugenia Leonova ${ }^{1}$ and \\ Ekaterina Stolyarevich 2,4 \\ 1 Nephrology, Botkin Hospital, 125284 Moscow, Russia; ansezy@gmail.com (A.Z.); \\ tmakarova24@gmail.com (T.M.); janeleonova999@gmail.com (E.L.) \\ 2 Nephrology, Moscow State University of Medicine and Dentistry, 127473 Moscow, Russia; \\ Stolyarevich@yandex.ru \\ 3 Faculty of Medicine, Lomonosov Moscow State University, 119991 Moscow, Russia \\ 4 Pathology, Moscow City Nephrology Center, 123182 Moscow, Russia \\ * Correspondence: helena.zakharova@gmail.com; Tel.: +7-9671346936
}

Citation: Zakharova, E.; Zykova, A.; Makarova, T.; Leonova, E.; Stolyarevich, E. Kidney Pathology and Outcomes in ANCA-Associated Vasculitis: Retrospective Analysis of 85 Patients. Kidney Dial. 2021, 1 , 61-73. https://doi.org/10.3390/ kidneydial1010010

Academic Editor: Giorgina Barbara Piccoli

Received: 26 June 2021

Accepted: 12 August 2021

Published: 22 August 2021

Publisher's Note: MDPI stays neutral with regard to jurisdictional claims in published maps and institutional affiliations.

Copyright: (C) 2021 by the authors. Licensee MDPI, Basel, Switzerland. This article is an open access article distributed under the terms and conditions of the Creative Commons Attribution (CC BY) license (https:/ / creativecommons.org/licenses/by/ $4.0 /)$.

\begin{abstract}
ANCA-associated vasculitis (AAV) poses a significant risk of kidney failure; kidney biopsy remains a key prognostic tool. The histopathologic classification of AAV glomerulonephritis (GN) developed by Berden et al. showed correlation between GN classes and kidney outcomes; ANCA Renal Risk Score (ARRS) included tubular atrophy and interstitial fibrosis (TA/IF) as an additional parameter for risk assessment. We aimed to evaluate kidney survival across AAV GN classes and ARRS groups. A single-center retrospective study included 85 adult patients with biopsy-proven AAV kidney disease followed in the period of 2000-2020. Primary outcome was kidney survival at the end of $18(5 ; 66)$ months follow-up, and kidney death was considered as stage 5 CKD. We found significant differences in kidney survival for sclerotic, mixed, crescentic and focal AAV GN classes: $19 \%, 76.2 \%, 91.7 \%$ and $100 \%$, respectively ( $p=0.009$ ). Kidney survival was $0 \%, 75.6 \%$ and $100 \%$ for the high-, medium- and low-risk ARRS groups, respectively $(p<0.001)$; TA/IF analysis showed kidney survival at $49.6 \%$ vs. $87.7 \%$ for widespread and mild TA/IF, respectively $(p=0.003)$. Kidney survival was significantly lower in anti-MPO-ANCA versus anti-PR3-ANCA carriers $(50.3 \%$ and $78.1 \%$, respectively, $p=0.045$ ). We conclude that unfavorable AAV kidney outcomes are associated with sclerotic GN class by Berden's classification, ARRS high risk group, and anti-MPO-ANCA subtype.
\end{abstract}

Keywords: microscopic polyangiitis; granulomatosis with polyangiitis; eosinophilic granulomatosis with polyangiitis; kidney biopsy; pauci-immune focal and segmental necrotizing and crescentic glomerulonephritis; tubular atrophy and interstitial fibrosis; kidney survival

\section{Introduction}

ANCA-associated vasculitis (AAV) poses a significant risk of kidney failure; frequency of kidney involvement in AAV varies from 90\% in Microscopic Polyangiitis (MPA) and 80\% in Granulomatosis with Polyangiitis (GPA) to 45\% in Eosinophilic Granulomatosis with Polyangiitis (EGPA) [1,2]. Therefore, kidney survival remains one of the most important outcomes in AAV. A systematic review of 44 studies, performed in 2008 by the European Vasculitis Study Group, found valuable data for GPA only, with kidney survival at 93\% and $85 \%$ for 1 year and 5 years, respectively; the data for MPA and EGPA were scarce. [3]. Eight years later, a multicenter study of 144 patients with MPA managed according to the EULAR recommendations [4] showed that stage 5 chronic kidney disease (CKD) developed in $7.6 \%$ of patients with median follow-up of 33.5 months [5]. However, another study, including $80 \%$ of patients with MPA or renal-limited AAV, demonstrated less optimistic results- $47 \%$ of patients reached stage 5 CKD by the end of the 5-year follow-up period [6]. A study of the Asian population confirmed unfavorable kidney outcomes for MPA-30.1\% of patients reached a composite end-point (doubling of serum creatinine or stage 5 CKD) 
in 17 months, regardless of the immunosuppressive treatment [7]. In contrast, the most recent data concerning kidney survival in EGPA, provided by the retrospective study of 63 patients, show that just $17 \%$ of patients reached stage 5 CKD, with median follow-up of 48 months [8].

Evaluation of ANCA titers in association with kidney outcomes did not show a direct correlation between clinical remission and decrease in ANCA levels [9]; the role of ANCA subtype remains controversial. A review, published in 2016, concluded that the anti-proteinase3 (anti-PR3) subtype of ANCA was associated with higher risk of relapses [10]. Later, a single-center study of 85 patients with AAV confirmed that the anti-myeloperoxidase (anti-MPO) subtype of ANCA was associated with a lower risk for relapse, but with the worst patients and kidney survival rate; additional unfavorable prognostic factors included older age at the disease onset and male gender [11]. The most recent publication of a long-term observation of patients with AAV demonstrated that recurrent or persistent ANCA-positivity was associated with worst kidney outcomes, and a recurrent pattern of anti-MPO-AAV was associated with worsening kidney function [12].

Patients with AAV, especially those with severe kidney disease, remain at high risk of unfavorable outcomes, despite currently available effective treatments [2,9,13-16]. Even though the prognostic value of ANCA titer and subtype and several other biomarkers, as well as the role of treatment and therapeutic strategies, have been extensively studied in association with kidney outcomes over the last few decades, kidney biopsy remains a key diagnostic and prognostic tool, in particular for ANCA-negative and renal-limited AAV [17]. The characteristic kidney lesion in AAV is pauci-immune focal and segmental necrotizing and crescentic glomerulonephritis (GN); however, pathology features vary from mild focal segmental extracapillary proliferation to diffuse crescentic GN and interstitial granulomas or infiltrates $[18,19]$. Moreover, differential diagnosis between GPA, EGPA and MPA is based, above all, on the presence or absence of the granulomatous inflammation, as it is stressed in the statements of the Chapel-Hill Consensus Conference 2012 [20] as well as in the algorithms of the European Medical Agency (EMA) [21].

The histopathologic classification of AAV GN, proposed by Berden et al., demonstrated a correlation between GN classes and kidney outcomes [22]. This classification distinguishes four classes: focal ( $\geq 50 \%$ of normal glomeruli), extracapillary or crescentic $(\geq 50 \%$ of the glomeruli with cellular crescents), sclerotic $(\geq 50 \%$ of totally sclerosed glomeruli), and mixed; interstitial damage did not show significant influence on the kidney failure rate, which initially led to the conclusion that glomerular damage plays a key role [22]. However, later, the same group showed that tubular atrophy and necrosis were significant predictors of the decline in kidney function [23]. In the last decade, several other histological classifications for renal AAV were proposed and validated [24-27]. Thus, the recently developed clinicopathologic ANCA Renal Risk Score (ARRS) for prediction of renal outcome in AAV GN includes tubular atrophy and interstitial fibrosis (TA/IF) as one of the parameters for the risk assessment. Proposed and validated parameters are the percentage of normal glomeruli (N: 0-4-6 points), percentage of TA/IF (T: 0-2 points), and glomerular filtration rate (GFR) (G: $0-3$ points); the calculated sum of points distinguishes three risk groups: low ( 0 points), medium ( $2-7$ points), and high (8-11 points) [28]. In addition, a higher percentage of normal glomeruli was found in the biopsies of patients with a comparable GFR level at the time of kidney biopsy and anti-PR3 subtype versus anti-MPO subtype. Anti-MPO-ANCA was also associated with the higher extent of fibrotic lesions-both glomerulosclerosis and interstitial fibrosis [29,30].

In this retrospective study of AAV kidney outcomes, we aimed to evaluate kidney survival in association with GN class according to Berden's classification, with the ARRS group, and with the ANCA subtypes. 


\section{Materials and Methods}

A single-center retrospective study, using an electronic database, identified 121 patients $\geq 18$ years old with $\mathrm{AAV}$, diagnosed in our center in the period of 2000-2020 according to the ACR [31] criteria and EMA algorithm [21]. For the purpose of this study, the main data retrieved were age, gender, main diagnosis, systemic signs and symptoms and serum creatinine at presentation, disease activity, ANCA levels and subtype, date of kidney biopsy, kidney pathology findings, initial and subsequent treatment regimens, follow-up duration, and eGFR at the end of the follow-up.

Ultrasound-guided kidney biopsy was performed according to the standard inhospital protocol, with specimen handling and pathology evaluation in the dedicated kidney pathology unit. For light microscopy, sections of formalin-fixed paraffin-embedded tissue were stained with H\&E, Masson's trichrome, periodic acid-Schiff and Congo Red. Immunofluorescence was performed on frozen sections with FITS-conjugated antibodies to IgA, IgG, IgM, C1q, C3, fibrinogen, lambda and kappa light chains. ANCA levels were tested using immunoenzyme assay or indirect immunofluorescence. Disease activity was scored at the time of diagnosis, based on the Birmingham Vasculitis Activity Index (BVAS v.3) [32]. Pattern and severity of kidney disease were evaluated according to Berden's classification and ARRS [22,28].

After evaluation of clinical, serology and pathology findings, 36 patients with dual phenotype (both ANCA and anti-GBM positivity) or with AAV, superimposed on IgAvasculitis, and cases without adequate kidney pathology data (less than 5 glomeruli in the biopsy specimen) were excluded from further analysis (Figure 1). The median number of glomeruli per specimen in the study group was 13 (12; 19); 82 out of 85 cores contained $\geq 10$ glomeruli, and only 3 cores contained 7 and 6 glomeruli, respectively. The study group comprised 85 patients with pathology-proven AAV kidney disease, including $51(60 \%)$ females and $34(40 \%)$ males, with a median age of $55.5(41 ; 61.7)$ years. GPA was diagnosed in $34(40 \%)$ patients, MPA in $44(51.8 \%)$ patients, and EGPA in $7(8.2 \%)$ patients.

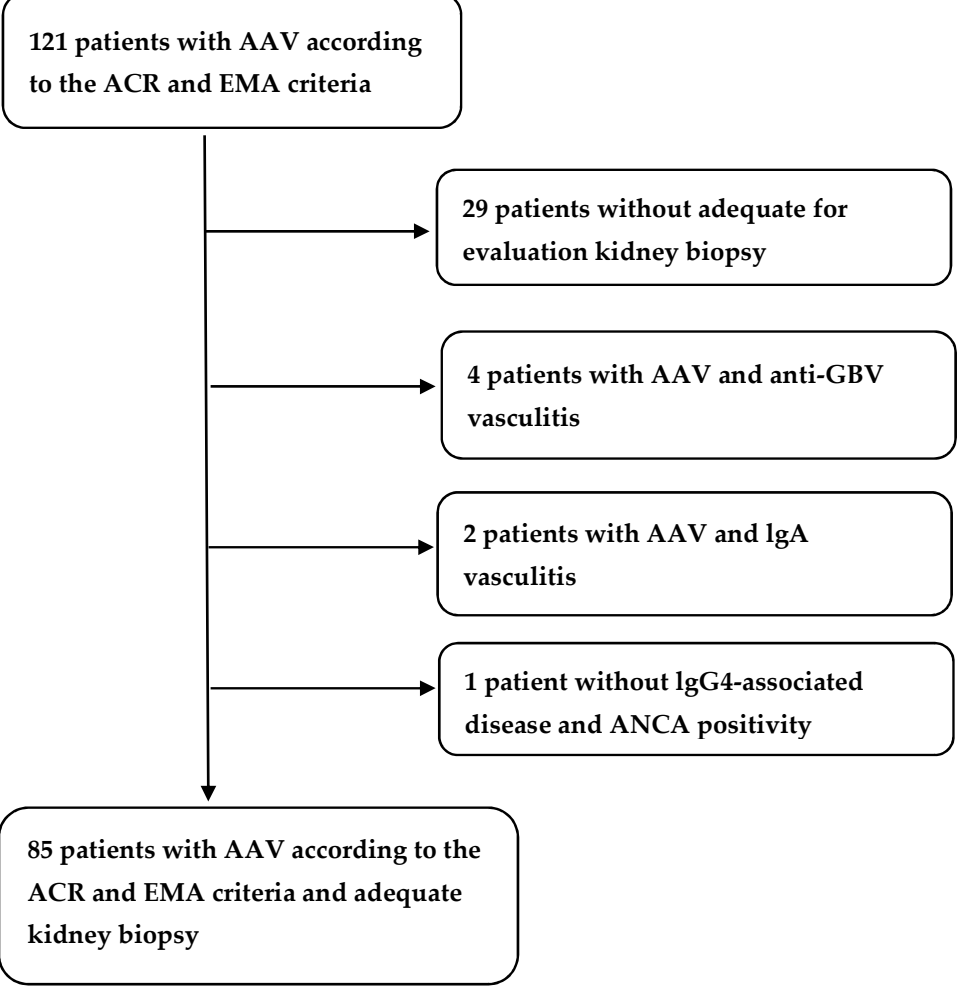

Figure 1. Study group selection. 
Initial treatment regimens included glucocorticoids plus intravenous cyclophosphamide in $57(67 \%)$ cases, glucocorticoids plus rituximab in $12(14.1 \%)$ cases, and glucocorticoids plus cyclophosphamide followed by rituximab in $16(18.8 \%)$ cases. Seven patients presented with pulmonary hemorrhage and received plasma exchanges. Subsequent treatment regimens included low doses of glucocorticoids combined with azathioprine or methotrexate for patients who initially received cyclophosphamide. Patients who received rituximab for initial treatment continued on rituximab.

Primary outcome was kidney death at the end of follow-up. The end of follow-up was defined as the date of the last visit or the date of the patient's death. Kidney death was considered as stage 5 CKD (eGFR $<15 \mathrm{~mL} / \mathrm{min} / 1.73 \mathrm{~m}^{2}$ CKD-EPI equation), regardless of the demand for kidney replacement therapy.

The data were presented as number (percentage) for categorical variables and median (interquartile range (IQR)) for continuous variables. Spearman's rank correlation coefficient was used for statistical correlations. Kidney survival rate was assessed using the KaplanMeier method. Differences between categories were assessed using the log-rank test; $p$ value $<0.05$ was considered as significant. The data analysis was performed on a personal computer using the statistical package IBM SPSS Statistics v 23.

\section{Results}

\subsection{Main Disease Characteristics}

Elevated ANCA levels found in 77 (90.6\%) of patients, 42 of which had anti-MPOANCA and 25-anti-PR3-ANCA; in 10 cases, a screening test for ANCA using indirect immunofluorescence was performed without identification of ANCA subtype; 8 patients $(9.4 \%)$ had seronegative AAV. All patients had severe disease with a median BVAS score of $15(12.3 ; 19.8)$ at the time of diagnosis. Systemic features and kidney pathology are shown in Table 1.

Table 1. Clinical manifestations and kidney pathology.

\begin{tabular}{|c|c|c|c|c|c|}
\hline Systemic Features & All Patients (85) & $\begin{array}{c}\text { Anti-PR3-Positive } \\
\text { (25) }\end{array}$ & $\begin{array}{l}\text { Anti-MPO-Positive } \\
(42)\end{array}$ & $\begin{array}{l}\text { ANCA Screening } \\
\text { Test-Positive (10) }\end{array}$ & $\begin{array}{c}\text { Sero-Negative } \\
\text { (8) }\end{array}$ \\
\hline \multicolumn{6}{|l|}{ Lungs, N (\%) } \\
\hline $\begin{array}{l}\text { Interstitial lung } \\
\text { damage }\end{array}$ & $22(25.9)$ & $8(32)$ & $3(30.9)$ & $2(20)$ & $0(0)$ \\
\hline Infiltrates & $16(18.8)$ & $3(12)$ & 8 (19) & $4(40)$ & $1(12.5)$ \\
\hline Cavities & $3(3.5)$ & $3(12)$ & $0(0)$ & $2(20)$ & $0(0)$ \\
\hline Nodular masses & $3(3.5)$ & $2(8)$ & $1(2.4)$ & 0 & $1(12.5)$ \\
\hline Asthma & $7(8.2)$ & $2(8)$ & $4(9.5)$ & $1(10)$ & $2(25)$ \\
\hline $\begin{array}{l}\text { Hemoptysis, } \\
\text { pulmonary } \\
\text { hemorrhage }\end{array}$ & $11(12.9)$ & $3(12)$ & $6(14.3)$ & $1(10)$ & $1(12.5)$ \\
\hline Skin, N (\%) & $22(25.8)$ & $6(24)$ & $16(38.1)$ & $2(20)$ & $1(12.5)$ \\
\hline Joints, N (\%) & $27(31.7)$ & $11(44)$ & $12(28.6)$ & $2(20)$ & $2(25)$ \\
\hline $\begin{array}{c}\text { Gastrointestinal, } \mathrm{N} \\
(\%)\end{array}$ & $6(7.0)$ & $2(8)$ & $3(7.1)$ & $1(10)$ & $1(12.5)$ \\
\hline $\begin{array}{c}\text { Nervous system, } \mathrm{N} \\
(\%)\end{array}$ & $12(14.1)$ & $6(24)$ & $5(11.9)$ & 0 & $1(12.5)$ \\
\hline ENT, N (\%) & $38(44.7)$ & $19(76)$ & $16(38.1)$ & $2(20)$ & $4(50)$ \\
\hline Eyes, N (\%) & $13(15.3)$ & $8(32)$ & $4(9.5)$ & $0(0)$ & $1(12.5)$ \\
\hline $\begin{array}{l}\text { BVAS v.3 at } \\
\text { presentation }\end{array}$ & $\begin{array}{c}15.0 \\
(12.9 ; 19.8)\end{array}$ & $\begin{array}{c}15 \\
(14 ; 20)\end{array}$ & $\begin{array}{c}15 \\
(13 ; 20)\end{array}$ & $\begin{array}{c}14 \\
(13 ; 18.5)\end{array}$ & $\begin{array}{c}18 \\
(11 ; 20.5)\end{array}$ \\
\hline
\end{tabular}


Table 1. Cont.

\begin{tabular}{|c|c|c|c|c|c|}
\hline Systemic Features & All Patients (85) & $\begin{array}{c}\text { Anti-PR3-Positive } \\
\text { (25) }\end{array}$ & $\begin{array}{l}\text { Anti-MPO-Positive } \\
\text { (42) }\end{array}$ & $\begin{array}{l}\text { ANCA Screening } \\
\text { Test-Positive (10) }\end{array}$ & $\begin{array}{c}\text { Sero-Negative } \\
\text { (8) }\end{array}$ \\
\hline $\begin{array}{l}\text { Median serum } \\
\text { creatinine at } \\
\text { presentation, } \\
\mu \mathrm{mol} / \mathrm{L}\end{array}$ & $\begin{array}{c}257.0 \\
(171.0 ; 528.5)\end{array}$ & $\begin{array}{c}203 \\
(130 ; 311)\end{array}$ & $\begin{array}{c}139 \\
(120 ; 347)\end{array}$ & $\begin{array}{c}250 \\
(151.5 ; 576)\end{array}$ & $\begin{array}{c}100 \\
(73 ; 308.5)\end{array}$ \\
\hline \multicolumn{6}{|l|}{$\begin{array}{c}\text { Kidney pathology } \\
\text { (Berden's } \\
\text { classification) }\end{array}$} \\
\hline Focal, N (\%) & $20(23.5)$ & $3(12)$ & $11(26.2)$ & $3(30)$ & $3(37.5)$ \\
\hline Crescentic, N (\%) & $22(25.9)$ & $8(32)$ & $9(21.4)$ & $3(30)$ & $2(25)$ \\
\hline Mixed, N (\%) & $18(21.2)$ & $7(28)$ & $9(21.4)$ & $2(20)$ & $0(0)$ \\
\hline Sclerotic, N (\%) & $24(28.2)$ & $7(28)$ & $12(28.6)$ & $2(20)$ & $3(37.5)$ \\
\hline \multicolumn{6}{|l|}{ ARRS } \\
\hline Low, N (\%) & $22(25.9)$ & $7(28)$ & $9(21.4)$ & $2(20)$ & $4(25)$ \\
\hline Medium, N (\%) & $42(49.4)$ & $12(48)$ & $21(50)$ & $6(60)$ & $3(37.5)$ \\
\hline High, N (\%) & $21(24.7)$ & $6(24)$ & $12(28.6)$ & $2(20)$ & $1(12.5)$ \\
\hline
\end{tabular}

Median serum creatinine at presentation was $257.0(171 ; 528.5) \mu \mathrm{mol} / \mathrm{L}$. According to Berden's classification, 20 patients had focal GN, 22-crescentic GN, 18-mixed GN, and 24-sclerotic GN class; a single patient with severe kidney and lung involvement had interstitial nephritis without glomerular damage. Median ARRS in the main study group was 4 points $(2.0 ; 6.0)$, while 21 patients had high risk, 42 patients-medium risk, and 22-low risk according to the ARRS.

Follow-up duration did not differ significantly between patients with GPA, MPA and EGPA. By the end of the follow-up period, $66(77.6 \%)$ patients achieved remission, 12 (14.1\%) patients with remission of extrarenal manifestations developed ESKD and received dialysis treatment, and $7(8.2 \%)$ patients did not achieve remission and died from extrarenal complications.

\subsection{Kidney Survival Analysis in the Main Study Group}

At the end of the follow-up with the median of $18(5 ; 66)$ months, patient survival was $89.5 \%$, and kidney survival- $75.4 \%$. Patients of more advanced age at disease onset had more advanced CKD stages by the end of the follow-up period $(\mathrm{r}=0.303 ; p=0.011)$. Comparison of kidney outcomes in 2000-2010 and 2011-2020 did not show significant differences; kidney death occurred in $26.6 \%$ and $23.6 \%$ of cases, respectively $\left(\chi^{2}=0.098\right.$, $p=0.754)$.

Analysis of the outcomes for AAV GN classes defined according to Berden's classification showed significant difference in the kidney survival between all four classes: $19 \%, 76.2 \%, 91.7 \%$ and $100 \%$ for sclerotic, mixed, crescentic and focal classes, respectively $(p=0.009)$ (Figure 2).

Among 57 patients who received cyclophosphamide followed by azathioprine or methotrexate, kidney survival was $33 \%, 75 \%, 86.7 \%$ and $100 \%$ for sclerotic, mixed, crescentic and focal classes, respectively $(p=0.004)$. In 12 patients who received rituximab only, kidney survival was $0 \%$ for sclerotic class and $100 \%$ for mixed, crescentic and focal classes $(p=0.015)$. In the patients switched from cyclophosphamide to rituximab, kidney survival was $62 \%$ for the sclerotic class, $0 \%$ for the mixed class, and $100 \%$ for the focal and crescentic classes $(p=0.033)$. 


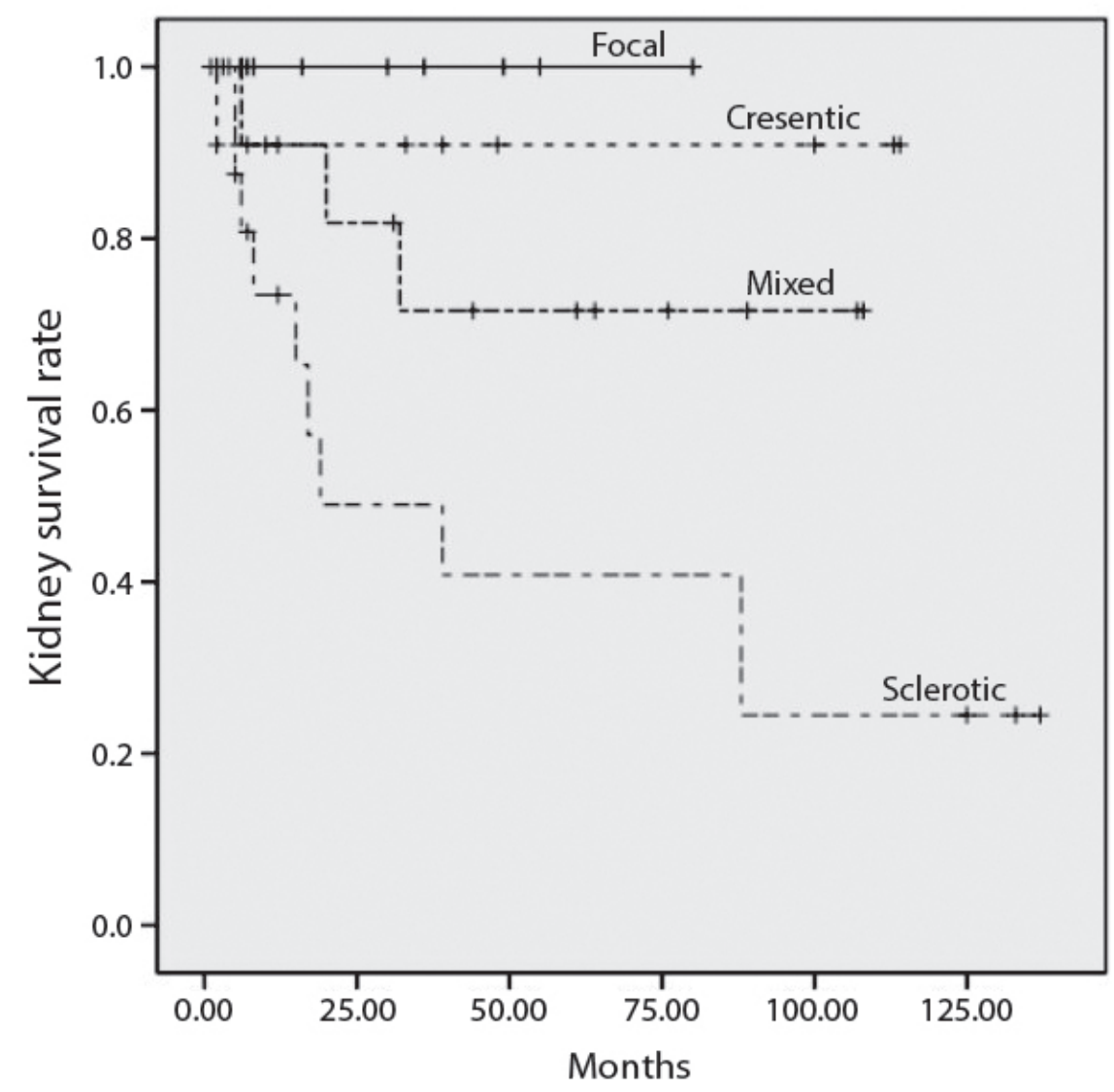

Figure 2. Kidney survival in the different classes of ANCA-associated glomerulonephritis according to Berden's classification $(p=0.009)$.

We found a statistically significant correlation between median ARRS at presentation and CKD stage at the end of follow-up $(r=0.490, p<0.001)$. The high ARRS group demonstrated unfavorable kidney outcome-kidney survival was $0 \%, 75.6 \%$ and $100 \%$ for the high, medium and low groups, respectively $(p<0.001)$ (Figure 3$)$.

In addition, kidney survival was $0 \%$ and $100 \%$ for the low- and high-risk group, respectively, regardless of the treatment regimen. In the medium-risk group, kidney survival was $100 \%$ in the patients who received rituximab only or were switched from cyclophosphamide to rituximab and continued on rituximab, and $81.8 \%$ in the patients who received cyclophosphamide followed by azathioprine or methotrexate $(p=0.438)$.

Using ARRS parameters, we evaluated the degree of TA/IF and of glomerular damage separately, and found that the patients with widespread TA/IF reached stage 5 CKD significantly more often compared to those with mild TA/IF: kidney survival was $49.6 \%$ vs. $87.7 \%$ for T2 and T0 points, respectively $(p=0.003)$ (Figure 4 ).

Similarly, the patients with a higher number of damaged glomeruli reached stage 5 CKD significantly more often, compared to those with a lower number of damaged glomeruli: kidney survival 30\%, $43.2 \%$ and $78.1 \%$ for N6, N4 and N0 points, respectively $(p=0.001)$ (Figure 5).

Finally, kidney survival was significantly lower in patients anti-MPO-ANCA compared to those with anti-PR3-ANCA ( $50.3 \%$ vs. $78.1 \%$, respectively, $p=0.045)$ (Figure 6). 


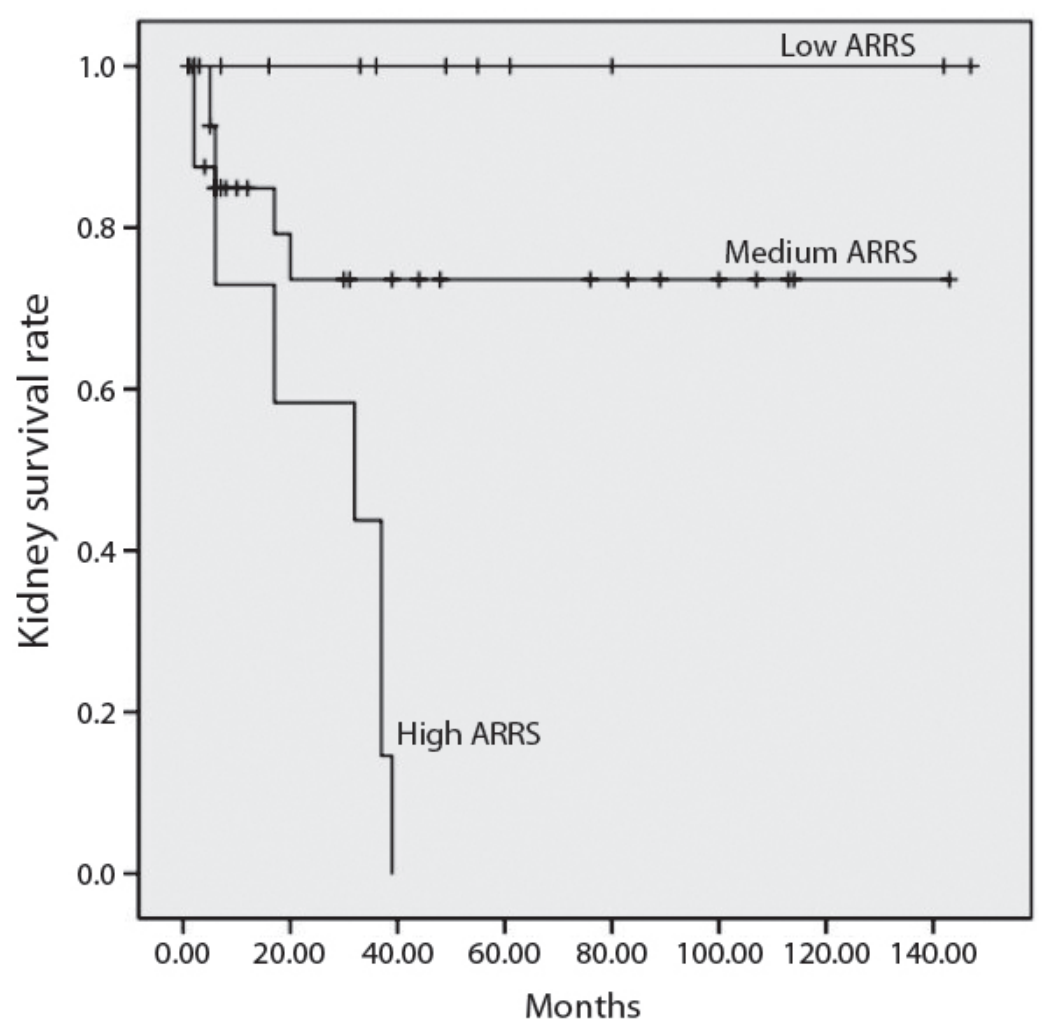

Figure 3. Kidney survival according to the ARRS group (Low, 0-1 points: Medium, 2-7 points: High, $8-11$ points) $(p<0.001)$.

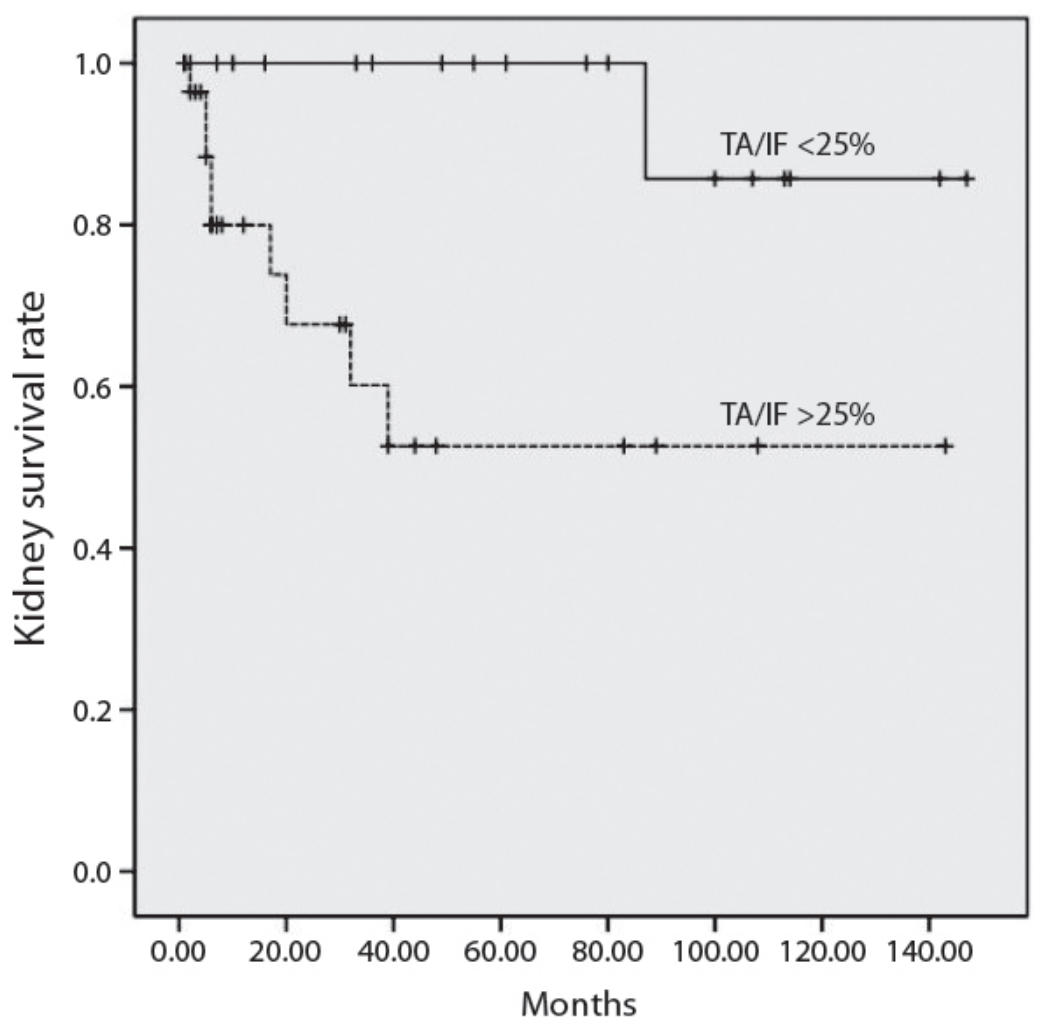

Figure 4. Kidney survival according to the tubular atrophy and interstitial fibrosis ARRS points $(\mathrm{T} 0<25 \%$; T2 $>25 \%)(p=0.003)$. 


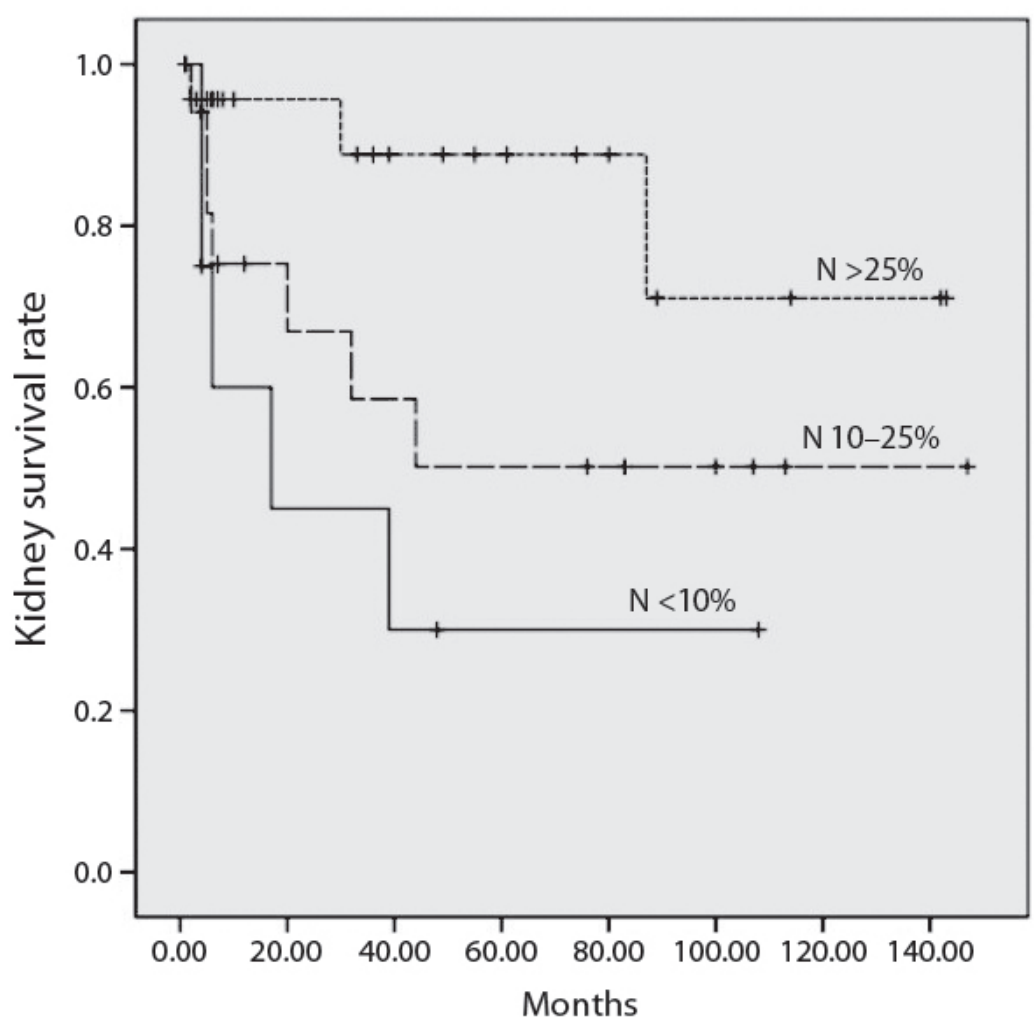

Figure 5. Kidney survival according to the percentage of normal glomeruli ARRS points (N0 > 25\%; N4 10-25\%; N6 < 10\%) ( $p=0.001)$.

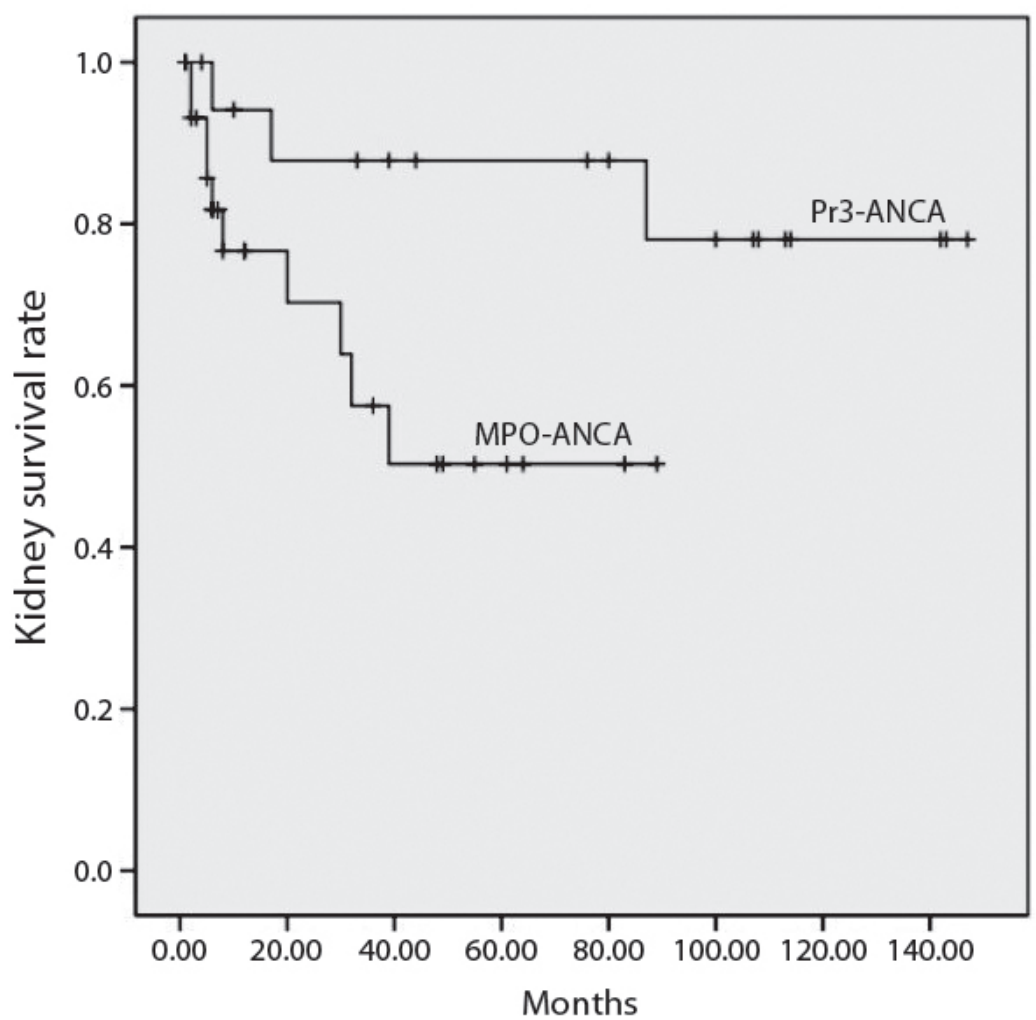

Figure 6. Kidney survival in the patients with anti-MPO-ANCA versus anti-PR3-ANCA $(p=0.045)$. 


\subsection{Kidney Survival Analysis in the Anti-PR3-ANCA and Anti-MPO-ANCA Subgroups}

Given the different renal outcomes in the patients with anti-PR3 and anti-MPO-ANCA in the main group, we divided patients with available ANCA subtype data into two subgroups-anti-MPO and anti-PR3 ANCA carriers. The results of analysis according to Berden's classification in the subgroups tend to be close to the general study group; however, we did not find statistically significant differences for all four classes in the particular subgroups. Kidney survival in the anti-PR3-ANCA subgroup was similarly high $(100 \%)$ for the crescentic and focal classes, and in the anti-MPO-ANCA subgroup-almost similarly low $(24.3 \%$ and $31 \%)$ for the sclerotic and mixed classes, respectively.

In contrast, analysis according to the ARRS showed statistically significant differences within the ANCA subtypes' subgroups. In the anti-PR3-ANCA subgroup, kidney survival was $0 \%, 82.5 \%$ and $100 \%(p=0.019)$ (Figure 7 ), and in the anti-MPO-ANCA subgroup, it was $0 \%, 41.8 \%$ and $100 \%(p=0.028)$ for the high, medium and low risk, respectively (Figure 8).

Kidney survival in the low- and high-ARRS risk groups did not differ significantly in both ANCA subtypes subgroups. However, the patients with medium ARRS risk and anti-MPO-ANCA had worse kidney survival compared to medium ARRS risk and antiPR3-ANCA ( $41.8 \%$ vs. $82.5 \%$, respectively), even though the differences did not reach statistical significance $(p=0.13)$.

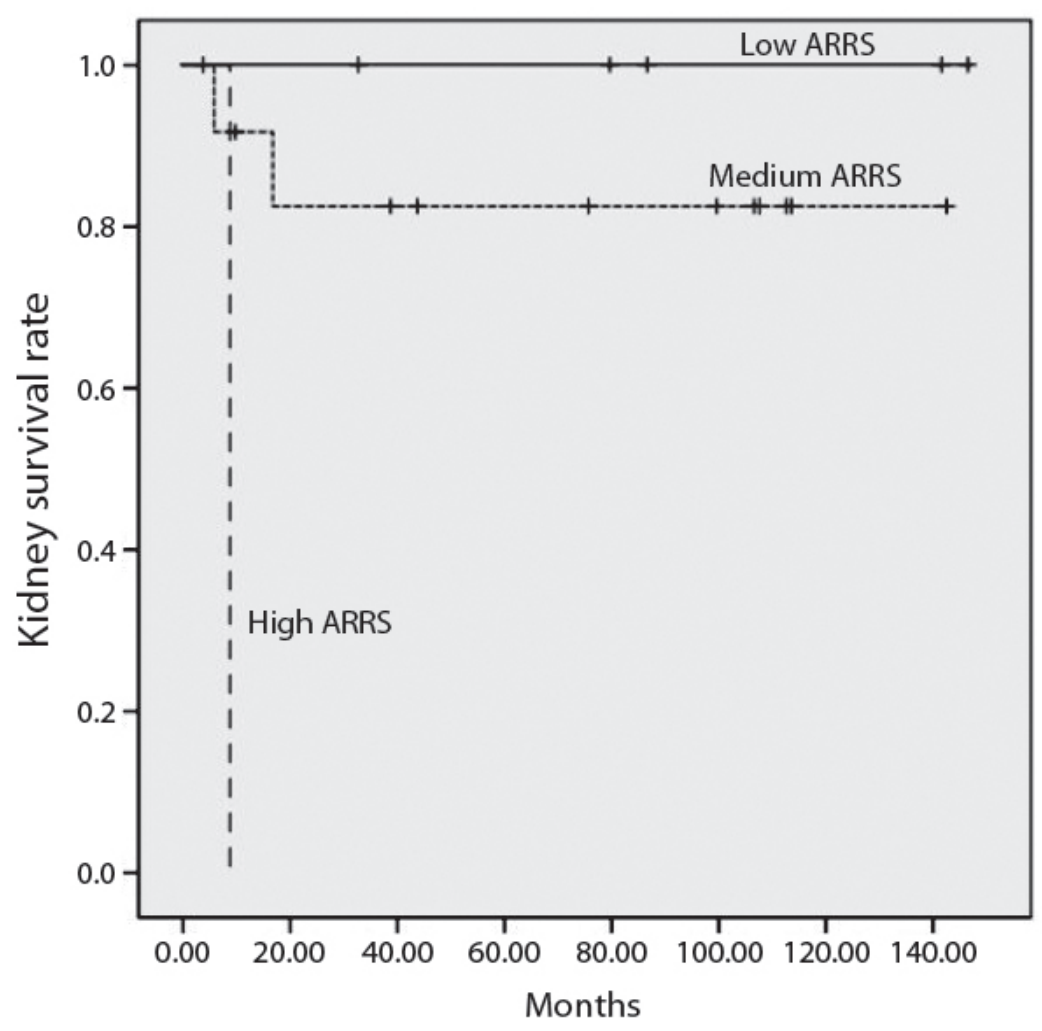

Figure 7. Kidney survival according to the ARRS score in the anti-PR3 subgroup $(p=0.019)$. 


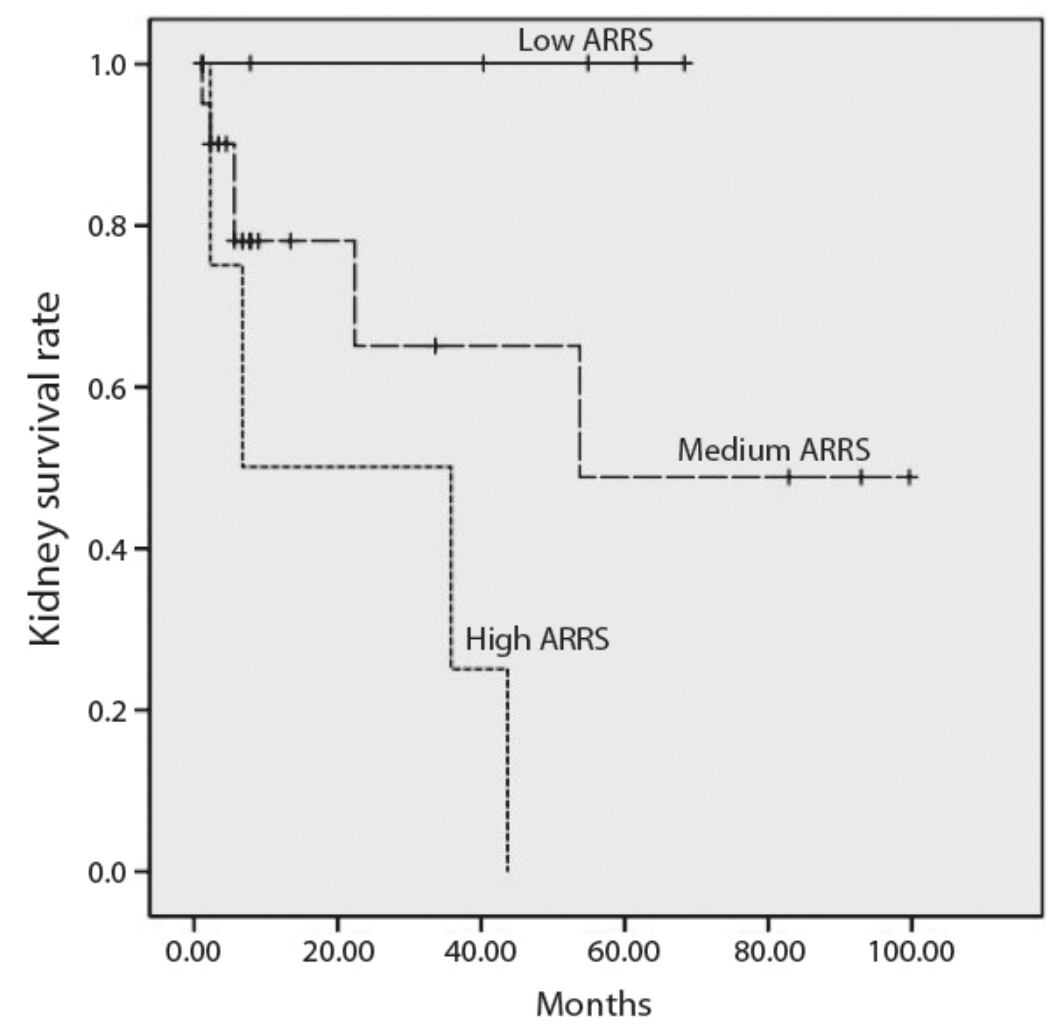

Figure 8. Kidney survival according to the ARRS score in the anti-MPO subgroup $(p=0.028)$.

\section{Discussion}

Patients with AAV remain at high risk of unfavorable outcomes despite currently available effective treatments [2-8]; evaluation of the prognostic factors for kidney outcomes at the time of diagnosis plays a key role. Kidney pathology not only confirms the diagnosis, but also predicts kidney survival and thus, guides the treatment strategy. Even though Berden's classification of the AAV GN clearly distinguishes focal and active lesions from diffuse and chronic, and demonstrates the impact of glomerular sclerosis on the kidney outcome, this classification does not accommodate TA/IF. However, the degree of tubulointerstitial damage and its role in CKD progression for glomerular diseases is well established [33-35] and the ARRS score's main advantage is incorporation of both glomerular and tubulointerstitial lesions.

In our study group, kidney survival by the end of the follow-up of $18(5 ; 66)$ months was $75.4 \%$, which is comparable to the most recent data [16]. We used both Berden's classification and ARRS to evaluate the prognostic value of kidney pathology. Our results are consistent with Berden's original publication data [22]: in our study group, the focal class, as expected, had the best outcome, and sclerotic class-the worst outcome. Of note, not all validation studies confirmed the difference between crescentic and mixed classes; thus, a large meta-analysis, including 1601 patients, did not show significant differences in the progression to stage 5 CKD between these classes (RR 1.14, 95\% CI 0.91-1.43, $p=0.27$ ) [36]. In our study, kidney survival in the crescentic class turned out significantly better compared to the mixed class in the general group, confirmed by the analysis in the anti-MPO-ANCA and anti-PR3 subgroups. Importantly, we found significantly worse kidney survival in the anti-MPO-ANCA carriers, regardless of kidney pathology findings, which confirms the negative impact of anti-MPO-ANCA on kidney outcomes [18,19,29,30,37]. Comparison of kidney survival across Berden's classification and according to treatment regimens did not show significant differences between the main study group and the group of patients who received initial treatment with cyclophosphamide. The results showed $19 \%, 76.2 \%$, $91.7 \%$ and $100 \%$ for the sclerotic, mixed, crescentic and focal classes, respectively, for the 
main group and $33 \%, 75 \%, 86.7 \%$ and $100 \%$ for the sclerotic, mixed, crescentic and focal classes, respectively, for the cyclophosphamide subgroup. However, the patients who received rituximab or were switched from cyclophosphamide to rituximab due to a lack of cyclophosphamide efficacy demonstrated $100 \%$ kidney survival not only for the focal, but also for the crescentic class, which means that rituximab may be more effective than cyclophosphamide for the treatment of active lesions.

The results of ARRS analysis in our study are also consistent with the original publication [28] data; we found that kidney survival in the high ARRS group was not surprisingly the worst. It is worth noting that the separate evaluation of glomerular and tubulointerstitial lesions confirmed significant differences in kidney survival depending on both $\mathrm{N}$ (percentage of normal glomeruli) and T (percentage of TA/IF) parameters. There are some data concerning isolated tubulointerstitial damage in AAV [38]; however, in our group, just one patient did not have glomerular lesions, which did not allow for analysis of this pattern. An additional finding in our study was the worse kidney survival within the medium ARRS risk group in the anti-MPO-ANCA carriers versus anti-PR3-ANCA carriers. The difference did not reach statistical significance, and the possible explanation refers to the data indicating that severe active lesions at the disease onset in anti-PR3 AAV may flatten the differences in prognostic value of ANCA subtypes [39,40]. However, summarizing the available data, Kronbichler and Jane [41] stressed the association of the anti-MPO-ANCA subtype with TA/IF, tubular necrosis, and glomerulosclerosis. Importantly, kidney survival in our study did not depend on the treatment regimen across the ARRS groups, confirming that ARRS, which includes tubulointerstitial damage and eGFR, is the most effective tool for prediction of kidney outcomes.

\section{Conclusions}

Our study confirms that unfavorable renal outcomes for AAV kidney disease are associated with sclerotic class according to Berden's classification of AAV GN, high ARRS risk group, and the presence of anti-MPO-ANCA; and demonstrates the strength of ARRS for selected groups of patients.

Author Contributions: Conceptualization, E.Z. and A.Z.; methodology, E.Z. and A.Z.; software, A.Z.; formal analysis, A.Z.; investigation, E.Z., T.M., E.L. and E.S.; resources, E.Z., E.S., T.M. and E.L.; data curation, E.Z. and A.Z.; writing — original draft preparation, A.Z.; writing—review and editing, E.Z.; visualization, A.Z.; supervision, E.Z. and E.S. All authors have read and agreed to the published version of the manuscript.

Funding: This research received no external funding.

Institutional Review Board Statement: Ethical review and approval were waived for this study, due to the retrospective nature of the study, not implying any prospective interventions.

Informed Consent Statement: Informed consent for kidney biopsy was obtained from all the patients prior to the procedure according to the in-hospital protocol. Patient consent for participation in this particular study was waived due to the retrospective nature of the study, not including any personal data.

Data Availability Statement: The data source is the patients' electronic records in the nephrology and kidney pathology units' databases, which contain personal information and thus cannot be shared.

Acknowledgments: We thank Elena Tareeva, Olga Vinogradova, Elena Nikitina, Alina Anilina, Evgeny Sgutov and Sergey Loginov for their help in the diagnosis and treatment of the patients.

Conflicts of Interest: The authors declare no conflict of interest. 


\section{References}

1. Jennette, J.C.; Falk, R.J. Small-Vessel Vasculitis. N. Engl. J. Med. 1997, 337, 1512-1523. [CrossRef]

2. Flossmann, O.; Berden, A.; de Groot, K.; Hagen, C.; Harper, L.; Heijl, C.; Höglund, P.; Jayne, D.; Luqmani, R.; Mahr, A.; et al. Long-term patient survival in ANCA-associated vasculitis. Ann. Rheum. Dis. 2011, 70, 488-494. [CrossRef]

3. Mukhtyar, C.; Flossmann, O.; Hellmich, B.; Bacon, P.; Cid, M.; Cohen-Tervaert, J.W.; Gross, W.L.; Guillevin, L.; Jayne, D.; Mahr, A.; et al. Outcomes from studies of antineutrophil cytoplasm antibody associated vasculitis: A systematic review by the European League Against Rheumatism systemic vasculitis task force. Ann. Rheum. Dis. 2008, 67, 1004-1010. [CrossRef] [PubMed]

4. Mukhtyar, C.; Guillevin, L.; Cid, M.C.; Dasgupta, B.; De Groot, K.; Gross, W.; Hauser, T.; Hellmich, B.; Jayne, D.; Kallenberg, C.G.M.; et al. EULAR recommendations for the management of primary small and medium vessel vasculitis. Ann. Rheum. Dis. 2008, 68, 310-317. [CrossRef] [PubMed]

5. Schirmer, J.H.; Wright, M.; Vonthein, R.; Herrmann, K.; Nölle, B.; Both, M.; Henes, F.O.; Arlt, A.; Gross, W.L.; Schinke, S.; et al. Clinical presentation and long-term outcome of 144 patients with microscopic polyangiitis in a monocentric German cohort. Rheumatology 2016, 55, 71-79. [CrossRef] [PubMed]

6. Rhee, R.L.; Hogan, S.L.; Poulton, C.J.; McGregor, J.A.G.; Landis, J.R.; Falk, R.J.; Merkel, P.A. Trends in Long-Term Outcomes Among Patients with Antineutrophil Cytoplasmic Antibody-Associated Vasculitis with Renal Disease. Arthritis Rheumatol. 2016, 68, 1711-1720. [CrossRef]

7. Shi, J.; Shen, Q.; Chen, X.-M.; Du, X.-G. Clinical characteristics and outcomes in microscopic polyangiitis patients with renal involvement: A study of 124 Chinese patients. BMC Nephrol. 2019, 20, 339. [CrossRef]

8. Durel, C.-A.; Sinico, R.A.; Teixeira, V.; Jayne, D.; Belenfant, X.; Marchand-Adam, S.; Pugnet, G.; Gaultier, J.; Le Gallou, T.; TitecaBeauport, D.; et al. Renal involvement in eosinophilic granulomatosis with polyangiitis (EGPA): A multicentric retrospective study of 63 biopsy-proven cases. Rheumatology 2021, 60, 359-365. [CrossRef]

9. Jones, R.B.; Tervaert, J.W.C.; Hauser, T.; Luqmani, R.; Morgan, M.D.; Peh, C.A.; Savage, C.O.; Segelmark, M.; Tesar, V.; Van Paassen, P.; et al. Rituximab versus Cyclophosphamide in ANCA-Associated Renal Vasculitis. N. Engl. J. Med. 2010, 363, 211-220. [CrossRef]

10. Cornec, D.; Gall, E.C.-L.; Fervenza, E.C.-L.G.F.C.; Specks, D.C.U. ANCA-associated vasculitis—Clinical utility of using ANCA specificity to classify patients. Nat. Rev. Rheumatol. 2016, 12, 570-579. [CrossRef]

11. Salmela, A.; Törnroth, T.; Poussa, T.; Ekstrand, A. Prognostic Factors for Survival and Relapse in ANCA-Associated Vasculitis with Renal Involvement: A Clinical Long-Term Follow-Up Study. Int. J. Nephrol. 2018, 2018, 1-11. [CrossRef] [PubMed]

12. Oristrell, J.; Loureiro-Amigo, J.; Solans, R.; Valenzuela, M.P.; Monsálvez, V.; Segarra, A.; Amengual, M.J.; Marín, A.; Feijoo, C.; Tolosa, C. Relapse rate and renal prognosis in ANCA-associated vasculitis according to long-term ANCA patterns. Clin. Exp. Immunol. 2021, 203, 209-218. [CrossRef] [PubMed]

13. Booth, A.D.; Almond, M.K.; Burns, A.; Ellis, P.; Gaskin, G.; Neild, G.H.; Plaisance, M.; Pusey, C.D.; Jayne, D.R. Outcome of ANCA-associated renal vasculitis: A 5-year retrospective study. Am. J. Kidney Dis. 2003, 41, 776-784. [CrossRef]

14. Walsh, M.; Casian, A.; Floßmann, O.; Westman, K.; Höglund, P.; Pusey, C.; Jayne, D.R. Long-term follow-up of patients with severe ANCA-associated vasculitis comparing plasma exchange to intravenous methylprednisolone treatment is unclear. Kidney Int. 2013, 84, 397-402. [CrossRef]

15. Walsh, M.; Merkel, P.A.; Peh, C.A.; Szpirt, W.M.; Puéchal, X.; Fujimoto, S.; Hawley, C.M.; Khalidi, N.; Floßmann, O.; Wald, R.; et al. Plasma Exchange and Glucocorticoids in Severe ANCA-Associated Vasculitis. N. Engl. J. Med. 2020, 382, 622-631. [CrossRef]

16. Moura, M.C.; Irazabal, M.V.; Eirin, A.; Zand, L.; Sethi, S.; Borah, B.J.; Winters, J.L.; Moriarty, J.P.; Cartin-Ceba, R.; Berti, A.; et al. Efficacy of Rituximab and Plasma Exchange in Antineutrophil Cytoplasmic Antibody-Associated Vasculitis with Severe Renal Disease. J. Am. Soc. Nephrol. 2020, 31, 2688-2704. [CrossRef]

17. Kronbichler, A.; Kerschbaum, J.; Gründlinger, G.; Leierer, J.; Mayer, G.; Rudnicki, M. Evaluation and validation of biomarkers in granulomatosis with polyangiitis and microscopic polyangiitis. Nephrol. Dial. Transplant. 2015, 31, 930-936. [CrossRef] [PubMed]

18. Bajema, I.M.; Hagen, E.C.; Hansen, B.E.; Hermans, J.; Noel, L.H.; Waldherr, R.; Ferrario, F.; Van Der Woude, F.J.; Bruijn, J.A. The renal histopathology in systemic vasculitis: An international survey study of inter- and intra-observer agreement. Nephrol. Dial. Transplant. 1996, 11, 1989-1995. [CrossRef]

19. Bajema, I.M.; Hagen, E.; Van Der Woude, F.J.; Bruijn, J.A. Wegener's granulomatosis: A meta-analysis of 349 literary case reports. J. Lab. Clin. Med. 1997, 129, 17-22. [CrossRef]

20. Jennette, J.C.; Falk, R.J.; Bacon, P.A.; Basu, N.; Cid, M.C.; Ferrario, F.; Flores-Suarez, L.F.; Gross, W.L.; Guillevin, L.; Hagen, E.C.; et al. 2012 Revised International Chapel Hill Consensus Conference Nomenclature of Vasculitides. Arthritis Rheum. 2013, 65, 1-11. [CrossRef]

21. Abdulkader, R.; Lane, S.E.; Scott, D.G.I.; Watts, R.A. Classification of vasculitis: EMA classification using CHCC 2012 definitions. Ann. Rheum. Dis. 2013, 72, 1888. [CrossRef]

22. Berden, A.E.; Ferrario, F.; Hagen, E.C.; Jayne, D.R.; Jennette, J.C.; Joh, K.; Neumann, I.; Noël, L.-H.; Pusey, C.D.; Waldherr, R.; et al. Histopathologic Classification of ANCA-Associated Glomerulonephritis. J. Am. Soc. Nephrol. 2010, 21, 1628-1636. [CrossRef] [PubMed]

23. Berden, A.E.; Jones, R.; Erasmus, D.D.; Walsh, M.; Noël, L.-H.; Ferrario, F.; Waldherr, R.; Bruijn, J.A.; Jayne, D.R.; Bajema, I.M.; et al. Tubular Lesions Predict Renal Outcome in Antineutrophil Cytoplasmic Antibody-Associated Glomerulonephritis after Rituximab Therapy. J. Am. Soc. Nephrol. 2011, 23, 313-321. [CrossRef] [PubMed] 
24. Kristensen, T.; Gregersen, J.W.; Krag, S.R.; Ivarsen, P. The relation between histopathological classification and renal outcome, ANCA subtype and treatment regimens in ANCA-associated vasculitis. Clin. Exp. Rheumatol. 2016, 34 (Suppl. 97), 105-110.

25. Moroni, G.; Binda, V.; Leoni, A.; Raffiotta, F.; Quaglini, S.; Banfi, G.; Messa, P. Predictors of renal survival in ANCA-associated vasculitis. Validation of a histopatological classification schema and review of the literature. Clin. Exp. Rheumatol. 2015, 33, 56-63.

26. Sethi, S.; D’Agati, V.D.; Nast, C.C.; Fogo, A.B.; De Vriese, A.; Markowitz, G.S.; Glassock, R.J.; Fervenza, F.C.; Seshan, S.V.; Rule, A.; et al. A proposal for standardized grading of chronic changes in native kidney biopsy specimens. Kidney Int. 2017, 91, 787-789. [CrossRef] [PubMed]

27. Berti, A.; Gall, E.C.-L.; Cornec, D.; Moura, M.C.; Matteson, E.L.; Crowson, C.S.; Ravindran, A.; Sethi, S.; Fervenza, F.C.; Specks, U. Incidence, prevalence, mortality and chronic renal damage of anti-neutrophil cytoplasmic antibody-associated glomerulonephritis in a 20-year population-based cohort. Nephrol. Dial. Transplant. 2019, 34, 1508-1517. [CrossRef]

28. Brix, S.R.; Noriega, M.; Tennstedt, P.; Vettorazzi, E.; Busch, M.; Nitschke, M.; Jabs, W.J.; Özcan, F.; Wendt, R.; Hausberg, M.; et al. Development and validation of a renal risk score in ANCA-associated glomerulonephritis. Kidney Int. 2018, 94, 1177-1188. [CrossRef]

29. Hauer, H.A.; Bajema, I.M.; van Houwelingen, H.C.; Ferrario, F.; Noël, L.-H.; Waldherr, R.; Jayne, D.R.; Rasmussen, N.; Bruijn, J.A.; Hagen, E.C. Determinants of outcome in ANCA-associated glomerulonephritis: A prospective clinico-histopathological analysis of 96 patients. Kidney Int. 2002, 62, 1732-1742. [CrossRef]

30. Hilhorst, M.; Wilde, B.; Vriesman, P.V.B.; Van Paassen, P.; Tervaert, J.W.C. Estimating Renal Survival Using the ANCA-Associated GN Classification. J. Am. Soc. Nephrol. 2013, 24, 1371-1375. [CrossRef]

31. Fries, J.F.; Hunder, G.G.; Bloch, D.A.; Michel, B.A.; Arend, W.P.; Calabrese, L.H.; Fauci, A.S.; Leavitt, R.Y.; Lie, J.T.; Lightfoot, R.W.; et al. The American College of Rheumatology 1990 criteria for the classification of vasculitis: Summary. Arthritis Rheum. 2010, 33, 1135-1136. [CrossRef]

32. Mukhtyar, C.; Lee, R.; Brown, D.; Carruthers, D.; Dasgupta, B.; Dubey, S.; Flossmann, O.; Hall, C.; Hollywood, J.; Jayne, D.; et al. Modification and validation of the Birmingham Vasculitis Activity Score (version 3). Ann. Rheum. Dis. 2009, 68, 1827-1832. [CrossRef] [PubMed]

33. Lai, K.N.; Chan, L.Y.; Leung, J.C. Mechanisms of tubulointerstitial injury in IgA nephropathy. Kidney Int. 2005, 67, S110-S115. [CrossRef]

34. Nasr, S.H.; Collins, A.B.; Alexander, M.P.; Schraith, D.F.; Hernandez, L.H.; Fidler, M.E.; Sethi, S.; Leung, N.; Fervenza, F.C.; Cornell, L.D. The clinicopathologic characteristics and outcome of atypical anti-glomerular basement membrane nephritis. Kidney Int. 2016, 89, 897-908. [CrossRef]

35. Mariani, L.H.; Martini, S.; Barisoni, L.; Canetta, P.; Troost, J.P.; Hodgin, J.B.; Palmer, M.; Rosenberg, A.; Lemley, K.V.; Chien, H.-P.; et al. Interstitial fibrosis scored on whole-slide digital imaging of kidney biopsies is a predictor of outcome in proteinuric glomerulopathies. Nephrol. Dial. Transplant. 2018, 33, 310-318. [CrossRef]

36. Chen, Y.-X.; Xu, J.; Pan, X.-X.; Shen, P.-Y.; Li, X.; Ren, H.; Chen, X.-N.; Ni, L.-Y.; Zhang, W.; Chen, N. Histopathological Classification and Renal Outcome in Patients with Antineutrophil Cytoplasmic Antibodies-associated Renal Vasculitis: A Study of 186 Patients and Metaanalysis. J. Rheumatol. 2016, 44, 304-313. [CrossRef]

37. De Joode, A.A.E.; Sanders, J.S.F.; Stegeman, C.A. Renal Survival in Proteinase 3 and Myeloperoxidase ANCA-Associated Systemic Vasculitis. Clin. J. Am. Soc. Nephrol. 2013, 8, 1709-1717. [CrossRef] [PubMed]

38. Plafkin, C.; Zhong, W.; Singh, T. ANCA vasculitis presenting with acute interstitial nephritis without glomerular involvement. Clin. Nephrol. Case Stud. 2019, 7, 46-50. [CrossRef]

39. Franssen, C.F.; Gans, R.O.; Arends, B.; Hageluken, C.; ter Wee, P.M.; Gerlag, P.G.; Hoorntje, S.J. Differences between antimyeloperoxidase- and anti-proteinase 3-associated renal disease. Kidney Int. 1995, 47, 193-199. [CrossRef] [PubMed]

40. Wijngaarden, R.A.D.L.V.; Hauer, H.A.; Wolterbeek, R.; Jayne, D.R.; Gaskin, G.; Rasmussen, N.; Noël, L.-H.; Ferrario, F.; Waldherr, R.; Hagen, E.C.; et al. Clinical and Histologic Determinants of Renal Outcome in ANCA-Associated Vasculitis: A Prospective Analysis of 100 Patients with Severe Renal Involvement. J. Am. Soc. Nephrol. 2006, 17, 2264-2274. [CrossRef] [PubMed]

41. Kronbichler, A.; Jayne, D.R. ANCA Renal Risk Score: Is prediction of end-stage renal disease at baseline possible? Kidney Int. 2018, 94, 1045-1047. [CrossRef] [PubMed] 